\title{
Acute gastric dilatation and ischemia associated with portal vein gas caused by binge eating
}

Kwangwoo Nam, Hyun Deok Shin, and Jeong Eun Shin

Division of Gastroenterology, Department of Internal Medicine, Dankook University Hospital, Cheonan, Korea

Received: January 31, 2017

Revised : May 8, 2017

Accepted: August 14, 2017

\section{Correspondence to}

Kwangwoo Nam, M.D.

Tel: +82-41-550-7630

Fax: $+82-41-550-7050$

E-mail: nambag1108@gmail.com
A 33-year-old woman presented to the emergency department with severe abdominal pain. She had a history of binge eating due to wedding preparation-related stress. She had consumed heavy meals, for more than 3 hours, following which she developed severe epigastric pain. Initial abdominal radiograph revealed a massively distended stomach without perforation (Fig. $1 \mathrm{~A})$; therefore, rapid decompression with gastric lavage (about $6 \mathrm{~L}$ ) was performed for 1 hour. Abdominopelvic computed tomography (CT) showed distended stomach with diffuse intrahepatic portal vein gas (PVG) (Fig. 1B). The patient agreed to perform further evaluation, and esophagogastroduodenoscopy (EGD) revealed dark-colored mucosa and diffuse edema with shallow ulceration along the greater curvature of the stomach, suggesting severe ischemia (Fig. 2A). Empirical intravenous antibiotics and proton pump inhibitors were administered. The patient's condition gradually improved with conservative management (Fig. ${ }_{1 C)}$. On the 7th day, EGD showed improved gastric ischemia (Fig. $2 \mathrm{~B}$ ), and she was able to consume liquid diet. On the 13th day, CT showed no evidence of PVG (Fig. 1D) and the patient remained stable.

Gastric ischemia is rare owing to the abundance of collateral vessels of the stomach. However, in case of severe acute gastric dilatation (AGD), excessive intragastric pressure $(>20 \mathrm{cmH} 2 \mathrm{O})$
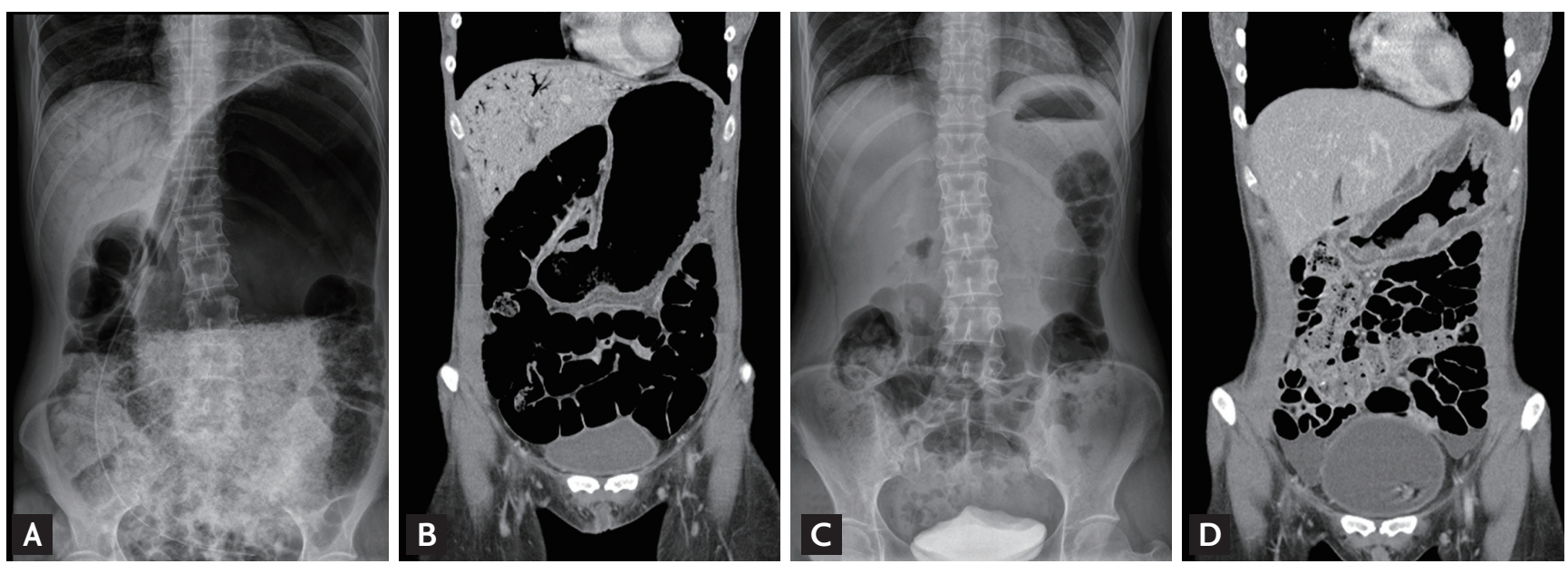

Figure 1. (A) Initial abdominal radiograph showing markedly distended stomach and intrahepatic portal vein gas (PVG). (B) Initial computed tomography (CT) scan taken after gastric lavage showing a reduction in size of the distended stomach and intrahepatic PVG. (C) Follow-up abdominal radiograph taken after gastric lavage showing significantly reduced size of the stomach. (D) Follow-up CT scan taken on the 13th day showing diffuse wall thickening of the stomach and absence of PVG. 

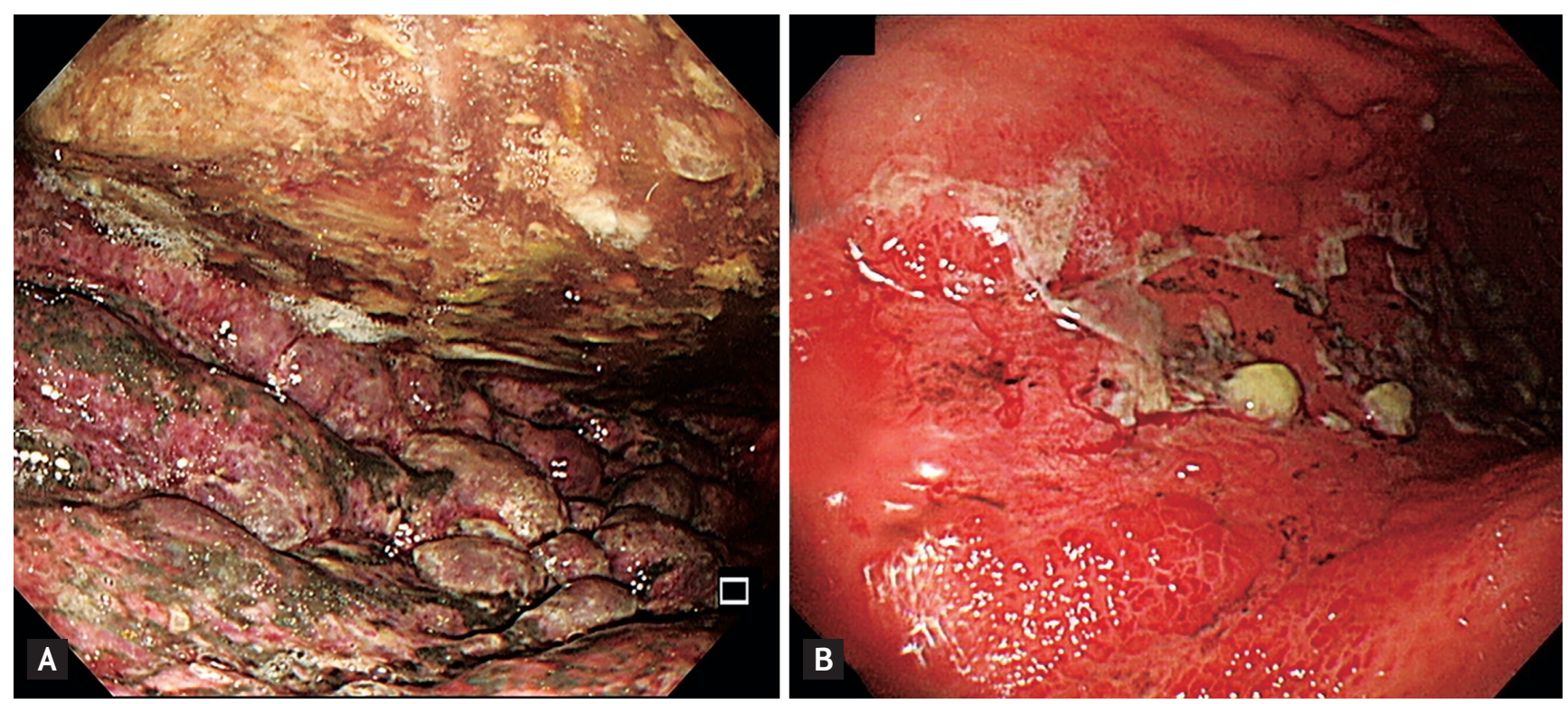

Figure 2. (A) Initial endoscopic image showing severely ischemic mucosal change along the greater curvature of the stomach. (B) Follow-up endoscopic image taken on the 7 th day showing improvement of gastric ischemia.

can block the intramural blood flow, resulting in gastric ischemia, necrosis, and perforation. AGD is commonly associated with eating disorder, psychiatric problems, or drug abuse, although it can occur in healthy patients. Immediate nasogastric decompression using a Levine tube is the mainstay of treatment. However, explorative laparotomy is required in some patients with clinical deterioration or frank perforation, which is associated with significant morbidity and mortality. PVG was caused by the migration of extensive intraluminal air due to AGD, and spontaneously disappeared in the follow-up CT. In conclusion, immediate gastric decompression is important in the management of AGD and can avert explorative laparotomy.

\section{Conflict of interest}

No potential conflict of interest relevant to this article was reported. 